Article

\title{
Emulsion Polymerization of Tung Oil-Based Latexes with Asolectin as a Biorenewable Surfactant
}

\author{
Ashley Johns, Kyle Edwards, Sarah Inglesby and Rafael L. Quirino * \\ Chemistry Department, Georgia Southern University, Statesboro, GA 30460, USA; \\ aj03037@georgiasouthern.edu (A.J.); ke01197@georgiasouthern.edu (K.E.); si00438@georgiasouthern.edu (S.I.) \\ * Correspondence: rquirino@georgiasouthern.edu; Tel.: +1-91-478-2345
}

Academic Editor: Alessandro Lavacchi

Received: 12 September 2016; Accepted: 1 November 2016; Published: 4 November 2016

\begin{abstract}
Bio-based vesicles, with potential application in drug delivery and/or catalyst encapsulation, have been prepared by the free radical emulsion co-polymerization of tung oil, divinylbenzene (DVB), $n$-butyl methacrylate (BMA), and asolectin in a xylene/water mixture. The free radical polymerization was initiated by di-tert-butyl peroxide (DTBP) at $100{ }^{\circ} \mathrm{C}$ in a convection oven. Molecular weights of approximately 11,000 Da were measured by Matrix-assisted Laser Desorption/Ionization-Time of Flight (Maldi-TOF) for tung oil-asolectin copolymers, verifying that significant polymerization occurs under the cure conditions employed. The cure of the co-monomer mixture employed in this work was monitored by Dielectric Analysis (DEA), while changes in the Raman spectrum of all co-monomers before and after the cure, along with differential scanning calorimetry (DSC) analysis, have been used to verify the need of a post-cure step and completion of the polymerization reaction. Scanning Transmission Electron Microscopy (STEM) images of the emulsion after polymerization indicate that vesicles were formed, and vesicle size distribution of samples prepared with different amounts of tung oil were determined using a Zetasizer.
\end{abstract}

Keywords: emulsion polymerization; tung oil; bio-based vesicles; AIBN; asolectin

\section{Introduction}

The development of bio-based materials as an alternative to traditional polymers and composites from non-renewable resources has gained significant attention in the past couple of decades due to economical and environmental factors. As a result, numerous vegetable oil-based thermosetting resin systems have been recently studied [1-6]. Vegetable oil-based resins recently developed include polyester amides [7], and cyanate esters [8]. Due to their abundance, low cost, readily availability, and renewable nature, vegetable oils have been used for the preparation of films, coatings, and thermosetting resins with interesting thermo-mechanical properties $[9,10]$.

With over $80 \%$ of its fatty acid chains bearing a conjugated triene, tung oil can react with vinyl co-monomers via cationic, thermal, or free radical polymerizations without the need of any structural modification of the triglyceride [9-11]. The resulting materials from the polymerization of tung oil consist of highly crosslinked thermosets. Whenever vegetable oil-based thermosetting resins are reinforced with materials exhibiting a polar surface, there is an intrinsic incompatibility between the hydrophobic resin and the hydrophilic reinforcement, which can be overcome by the addition of compatibilizers to the resin formulation [3,12]. Very recently, the successful use of asolectin as a natural compatibilizer for cellulose-reinforced tung oil-based composites has been reported [13].

Asolectin consists of a mixture of phospholipids from soybeans containing $76 \%$ of polyunsaturated fatty acids [14]. Indeed, the mixture contains approximately $25 \%$ of phosphatidylcholine, $25 \%$ of cephalin, and $25 \%$ of phosphatidylinositol (Figure 1), with the remainder consisting of small amounts of other phospholipids from soybeans [15]. Due to its amphiphilic nature, asolectin interacts with 
the non-polar resin components through its fatty acid chains and with the hydrophilic reinforcement through the phosphate and ammonium groups [13]. The polyunsaturated fatty acids in asolectin enable the phospholipid to be free radically crosslinked to other reactive and compatible olefins upon cure [13]. Due to obvious similarities in the structures of phospholipids and triglycerides, a copolymer of tung oil and asolectin is readily formed in the presence of a free radical initiator [13]. When cellulose/tung oil-based composites are prepared with asolectin, improved thermo-mechanical properties are obtained in comparison to composites prepared without asolectin [13].
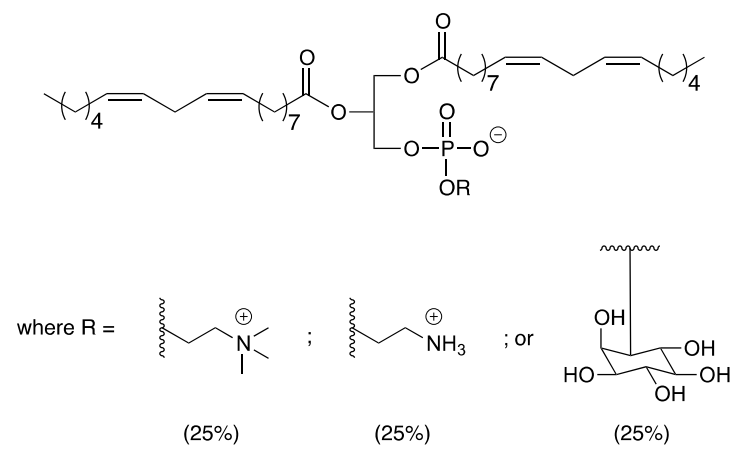

Figure 1. Chemical structure of asolectin.

The preparation of latexes and emulsions containing bio-based polymers allow for smooth film depositions leading to bio-based coatings [15,16]. Vegetable oil-based anionic [15], and cationic [16] polyurethane latexes with uniform particle sizes have been previously prepared. In these instances, the final polyurethane latexes were obtained by the co-polycondensation of vegetable oil-based polyols and diisocyanates in a water medium [15,16]. Polyurethane-acrylic hybrid systems have been developed with a similar concept [17]. In such systems, unreacted carbon-carbon double bonds from the vegetable oil-based polyols were crosslinked with acrylic co-monomers through free radical emulsion polymerization, resulting in hybrid latexes $[17,18]$. In such hybrid systems, the polyurethane forms a polar shell that serves as a high molecular weight emulsifier, while the vinyl polymers form a non-polar core [19].

Typically, emulsifiers are required in order to achieve successful free radical emulsion polymerization in solvent mixtures containing water [20]. In this work, asolectin is used for the first time as an effective bio-based emulsifier for the emulsion free radical co-polymerization of tung oil, divinylbenzene, and $n$-butyl methacrylate in a xylene/water mixture. The incorporation of asolectin in the crosslinked polymer results in stable bio-based polymeric vesicles with potential application in drug delivery and/or nano-catalyst encapsulation/stabilization. The successful free radical polymerization of xylene/water emulsions containing tung oil and asolectin was verified through Matrix-assisted Laser Desorption/Ionization-Time of Flight (Maldi-TOF). The cure of the monomer mixture was monitored by Dielectric Analysis (DEA), while changes in the Raman spectrum of all co-monomers before and after the reaction, along with differential scanning calorimetry (DSC) analysis, indicate the need of a post-cure step in order to attain completion of the polymerization reaction. Products obtained with different amounts of tung oil were further characterized by Thermogravimetric Analysis (TGA). The formation of vesicles was confirmed by Scanning Transmission Electron Microscopy (STEM) imaging, and vesicle size distribution was evaluated using a Zetasizer.

\section{Experimental}

\subsection{Materials}

Tung oil, asolectin from soybeans, di-tert-butyl peroxide (DTBP), and $n$-butyl methacrylate (BMA) were purchased from Sigma-Aldrich (St. Louis, MO, USA). Divinylbenzene (DVB) was acquired from 
TCI America (Portland, OR, USA), and xylenes and 3,5-dimethoxy-4-hydroxycinnamic acid were procured from Acros Organics (Pittsburg, PA, USA).

\subsection{Preparation of Asolectin-Containing Latexes}

Designated amounts of a resin containing $40 \mathrm{wt} . \%$ of tung oil, $30 \mathrm{wt} . \%$ of $n$-butyl methacrylate (BMA), $20 \mathrm{wt} . \%$ of DVB, and $10 \mathrm{wt} . \%$ of asolectin was initially prepared and added to a $20 \mathrm{~mL}$ scintillation vial. Whenever the percentage of tung oil in the mixture was changed as described in the text, a constant DVB:BMA ratio of 3:2 (by weight) was maintained. The percentage of asolectin was kept constant at $10 \mathrm{wt} . \%$ for all emulsions. The mixture was vortexed for $15 \mathrm{~s}$, leading to a seemingly homogenous liquid. After addition of an extra $5 \mathrm{wt} . \%$ of DTBP with respect to the total monomer mixture weight, the components were vortexed for another 15 s. $2.75 \mathrm{~g}$ of Xylene were then dispensed into the vial, followed by another $15 \mathrm{~s}$ of vortexing. Finally, $11.0 \mathrm{~g}$ of deionized water was added to the vial to create a 1:4 (xylene:water) by weight mixture. The vial was then capped, sealed with parafilm, and placed in a sonicator for $20 \mathrm{~min}$ to create an emulsion. After sonication, the vial was placed in a convection oven for $24 \mathrm{~h}$ at $100{ }^{\circ} \mathrm{C}$ for initial cure. After the initial curing process, the vial containing the emulsion was uncapped and dried for approximately $24 \mathrm{~h}$ in a vacuum oven at $60^{\circ} \mathrm{C}$. The dried contents were subjected to a post-cure step at $140{ }^{\circ} \mathrm{C}$ for $2 \mathrm{~h}$ in a convection oven to ensure complete polymerization, unless otherwise noted in the text.

\subsection{Characterization}

Maldi-TOF spectra were collected with a Microflex Maldi-TOF (Bruker Daltonics, Karlsruhe, Germany) using a linear method. Using 3,5-dimethoxy-4-hydroxycinnamic acid as the matrix, 1:50 (sample:matrix) by weight mixtures were prepared by mixing appropriate amounts of the ground post-cured sample and the matrix with a mortar and pestle. The dry sample/matrix mixture was sandwiched between two MSP 96 polished steel target plates and compressed at $1000 \mathrm{lbs}$ in a hydraulic press. The laser was set to 100 shots per run at $66 \%$ power and a frequency of $60 \mathrm{~Hz}$.

DSC experiments were conducted on a Q20 DSC instrument (TA Instruments, New Castle, DE, USA). Tests were run in a $\mathrm{N}_{2}$ atmosphere from $-20^{\circ} \mathrm{C}$ to $200^{\circ} \mathrm{C}$ at a heating rate of $10{ }^{\circ} \mathrm{C} / \mathrm{min}$ with sample sizes of $10 \mathrm{mg}$.

DEA experiments were conducted with an Epsilon DEA 230/1 cure monitor (Netzsch Instruments North America LLC, Burlington, MA, USA) to analyze the rate of cure. The instrument's probe was immersed in $10 \mathrm{~g}$ of the resin. The resin was cured in a convection oven held constantly at $100{ }^{\circ} \mathrm{C}$. The DEA test was conducted for $24 \mathrm{~h}$ with frequencies ranging from $0.1 \mathrm{~Hz}$ to $10,000.0 \mathrm{~Hz}$. The permittivity and loss factor were measured as a function of time, and the results displayed in the text convey a plot of ion viscosity $(\mathrm{Ohm} \cdot \mathrm{cm})$ versus time. The use of dielectric analysis for monitoring the polymerization of resins relies on the fact that in non-conductive polymers/substances, charge transfer occurs through the mobility of ions within the sample and is measured by the material's overall permittivity. As the polymerization progresses, longer chains are formed and ion mobility is gradually compromised, which is reflected in the materials permittivity. Once the polymerization is completed and polymer chain growth has ceased, no further change in ion mobility occurs and the permittivity remains constant. The ion viscosity discussed in in the text corresponds to a measurement of the resistance offered by a material to the transfer of a charge in a linear path. This property is measured in Ohm.cm and is referred to as "ion viscosity" due to the analogy with the flow of a substance through a medium.

Raman spectra were acquired with a DXR Raman Microscope (Thermo Fisher Scientific, Waltham, MA, USA). A total of 32 scans were collected per sample at an exposure time of $1 \mathrm{~s}$ for each scan. Laser power was set to $7.0 \mathrm{~mW}$, and a $50 \mu \mathrm{m}$ slit aperture was used.

A Q50 TGA instrument (TA Instruments, New Castle, DE, USA) measured weight loss of samples $(10 \mathrm{mg})$ under an air atmosphere as a function of temperature. Samples were heated from room temperature to $600{ }^{\circ} \mathrm{C}$ at a heating rate of $20^{\circ} \mathrm{C} / \mathrm{min}$. 
The morphology of the vesicles prepared was observed under Scanning Transmission Electron Microscopy mode (STEM) using a JEOL JSM-7600F field emission scanning electron microscope (Peabody, MA, USA) equipped with a transmission detector. The microscope was operated at $30.0 \mathrm{kV}$ accelerating voltage during STEM imaging. The suspension was deposited directly on a TEM copper grid after the heating treatment, and allowed to dry at ambient conditions for $24 \mathrm{~h}$ before imaging.

A nano series Zetasizer (Malvern Instruments, Worcestershire, UK) was used in order to obtain particle size and polydispersity index values. An aliquot of each emulsion was placed in a cuvette and diluted 10 times with deionized water. Measurements were made at $25^{\circ} \mathrm{C}$ with a $90^{\circ}$ angle of detection. Emulsion samples underwent three measurements with 11 runs per measurement for a duration of $10 \mathrm{~s}$ per run. Automatic attenuation selection was performed for each sample. The results reported in this manuscript correspond to the average of the three measurements carried out on each sample.

\section{Results and Discussion}

In order to verify that tung oil/asolectin-based emulsions can be polymerized under free radical conditions in the presence of DTBP, 1:4 (xylene:water) by weight emulsions of various ratios of tung oil and asolectin were cured in a convection oven at $100{ }^{\circ} \mathrm{C}$ for $24 \mathrm{~h}$. The $1: 4$ (xylene:water) by weight solvent ratio was determined based on related literature [20] and after a major screening of solvent combinations. As described previously, the polymerized emulsions were dried in a vacuum oven at $60{ }^{\circ} \mathrm{C}$ for $24 \mathrm{~h}$ and the dried materials recovered were subjected to Maldi-TOF analysis. Figure 2 shows the Maldi-TOF spectrum of the dry products from the emulsion containing a 1:1 weight ratio of tung oil and asolectin.

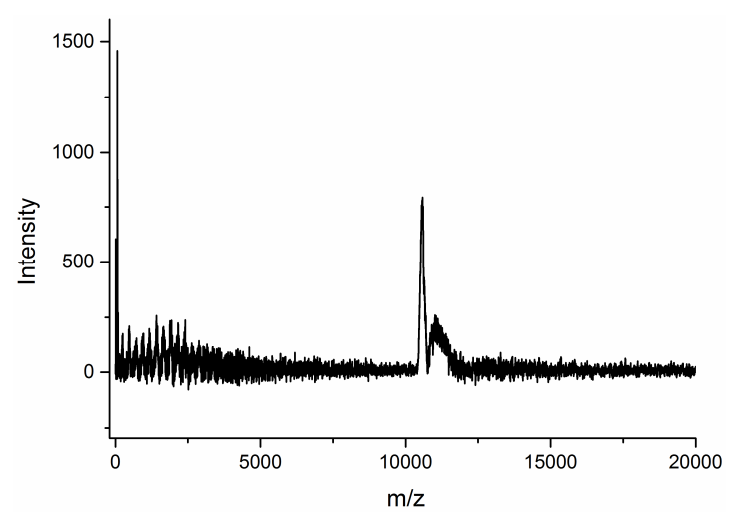

Figure 2. Maldi-TOF spectrum of dried products from a xylene/water emulsion containing a 1:1 weight ratio of tung oil and asolectin subjected to $24 \mathrm{~h}$ heating at $100{ }^{\circ} \mathrm{C}$ in the presence of di-tert-butyl peroxide (DTBP).

Two major signals can be clearly seen in the 11,000-12,000 m/z range in Figure 2, confirming the formation of high molecular weight products upon cure of the emulsion. Despite the promising results shown in Figure 2, the dry products obtained from tung oil and asolectin were exceedingly soft, rubbery, and tacky. In order to enhance the mechanical characteristics of the dry film, as demonstrated previously with related bio-based polyolefin systems [1], it became necessary to incorporate other reactive olefins, such as DVB and BMA. Upon addition of more reactive components, a natural increase of the dry products' crosslink density made it impossible to obtain clear Maldi-TOF spectra due to the inherent difficulty of analyzing thermoset materials through this technique.

Previously, the bulk free radical polymerization of $40 \mathrm{wt} . \%$ of tung oil, $30 \mathrm{wt} . \%$ of BMA, $20 \mathrm{wt} . \%$ of DVB, and $10 \mathrm{wt} . \%$ of asolectin had been successfully achieved after heating the crude resin in the presence of DTBP at $140{ }^{\circ} \mathrm{C}$ for $2 \mathrm{~h}$ and $50 \mathrm{~min}$ [13]. The same resin composition was therefore prepared into a xylene/water emulsion as described in the Experimental section. Xylene was chosen as the most appropriate solvent due to its compatibility with the monomers and also due to 
its relatively high boiling point, allowing for higher polymerization temperatures. Water has a lower boiling point than xylene, and therefore, the highest temperature at which the system can be cured is $100^{\circ} \mathrm{C}$. A weight ratio of 1:4 (xylene:water) was found to create a stable emulsion after $20 \mathrm{~min}$ of sonication. In order to select the optimum resin/solvent ratio, emulsions containing overall monomer weights of $200 \mathrm{mg}$, $400 \mathrm{mg}$, and $600 \mathrm{mg}$ were prepared, as shown in Figure 3.

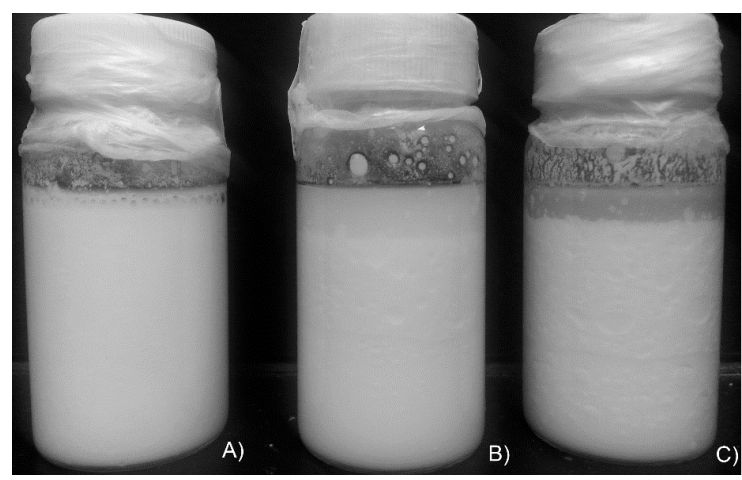

Figure 3. Emulsions of 1:4 (xylene:water) by weight containing (A) $200 \mathrm{mg}$ of total monomer weight, (B) $400 \mathrm{mg}$ of total monomer weight, and (C) $600 \mathrm{mg}$ of total monomer weight. The pictures have been taken immediately after the samples were prepared.

It is obvious from Figure 3 that an immediate phase separation occurs for samples containing $400 \mathrm{mg}$ (Figure 3B) and $600 \mathrm{mg}$ (Figure 3C) of total monomer weight, while a total monomer weight of $200 \mathrm{mg}$ (Figure 3A) produced no phase separation and therefore appeared to create a stable emulsion. The latter composition was observed over a period of $30 \mathrm{~min}$ in order to assess the stability of the emulsion formed. The results can be seen in Figure 4.

From the pictures in Figure 4, the emulsion containing $200 \mathrm{mg}$ of total monomer weight doesn't change its appearance for at least $30 \mathrm{~min}$ after preparation. It is therefore hypothesized that, if the free radical polymerization reaction starts within the first $30 \mathrm{~min}$ following the preparation of the emulsion, the monomers present in the non-polar micelles will start crosslinking before disruption of the emulsion, leading to spherical particles. The emulsions prepared held their stability for an extended period of time when kept in a closed container. No phase separation was observed since the emulsion preparation.

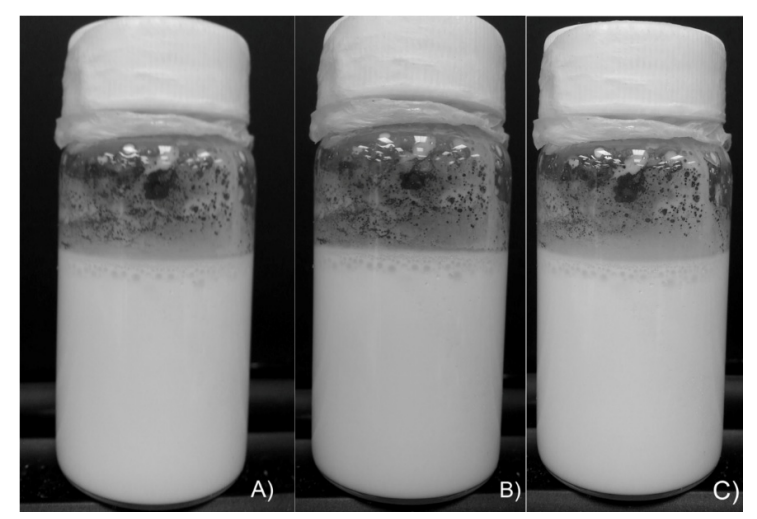

Figure 4. 1:4 (xylene:water) by weight emulsion containing $200 \mathrm{mg}$ of total monomer weight (A) immediately after sonication, (B) $15 \mathrm{~min}$ after sonication, and (C) $30 \mathrm{~min}$ after sonication.

The progress of the polymerization of the monomers at the cure temperature employed was monitored through dielectric analysis (DEA). The pure resin, containing $40 \mathrm{wt} . \%$ of tung oil, $30 \mathrm{wt} . \%$ of BMA, $20 \mathrm{wt} . \%$ of DVB, $10 \mathrm{wt} . \%$ of asolectin and an extra $5 \mathrm{wt} . \%$ of DTBP, was heated at $100{ }^{\circ} \mathrm{C}$ in a convection oven. The resulting DEA curve is displayed in Figure 5. 
In Figure 5, the initial decrease in ion viscosity is due to an increase in ion mobility as the monomers start heating. After $20 \mathrm{~min}$ of heating, ion viscosity begins to increase as the polymerization process begins. Based on the stability experiment results (Figure 4), the onset polymerization time of $20 \mathrm{~min}$ is ideal for the cure of emulsions since within that time frame, the emulsion will still be intact. The continuous increase in the slope of the DEA curve for the remainder of the experiment indicates a continuous progress in the polymerization process. Over the course of $24 \mathrm{~h}$, the DEA curve doesn't plateau, indicating that the resin isn't fully polymerized within the duration of the experiment. These results suggest that a post-cure step might be needed in order to complete the polymerization reaction.

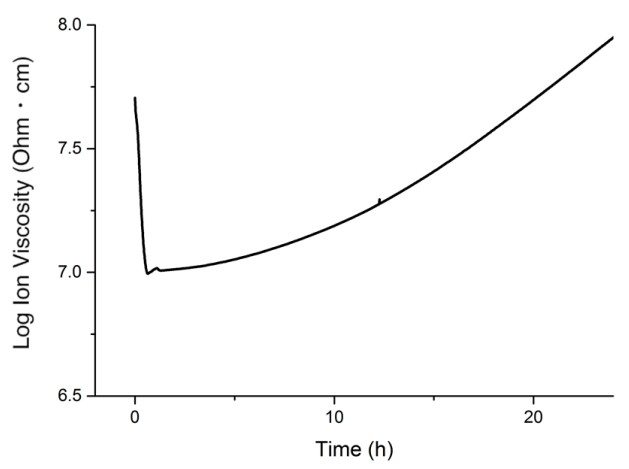

Figure 5. Dielectric Analysis curve of 40 wt.\% tung oil, 30 wt.\% $n$-butyl methacrylate (BMA), $20 \mathrm{wt} . \% \mathrm{DVB}$, and $10 \mathrm{wt} . \%$ asolectin heated at $100{ }^{\circ} \mathrm{C}$ for $24 \mathrm{~h}$.

In order to investigate the effect of tung oil concentration in the cure of tung oil/asolectin-based emulsions, samples with varying concentrations of tung oil were prepared and cured at $100{ }^{\circ} \mathrm{C}$ for $24 \mathrm{~h}$. Maintaining the concentration of asolectin constant at $10 \mathrm{wt} . \%$ and the BMA/DVB weight ratio set at 1.5, four samples containing $40 \mathrm{wt} . \%, 55 \mathrm{wt} . \%, 70 \mathrm{wt} . \%$, and $80 \mathrm{wt} . \%$ of tung oil were prepared and cured at $100{ }^{\circ} \mathrm{C}$ for $24 \mathrm{~h}$. The dried products from these samples were analyzed by DSC as shown in Figure 6.

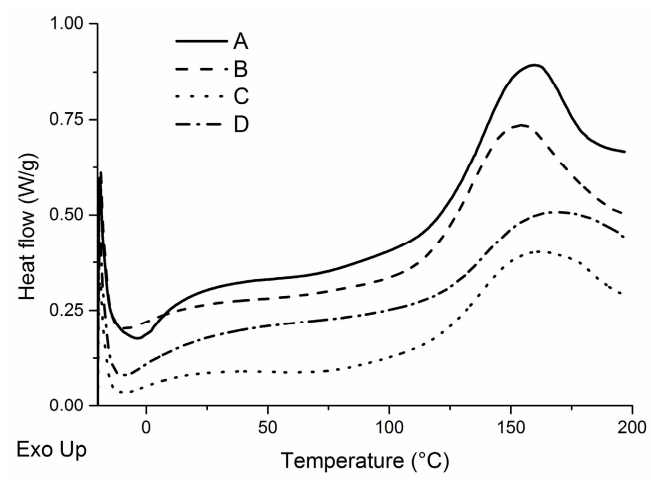

Figure 6. Differential Scanning Calorimetry results for dried products from emulsions containing (A) $80 \mathrm{wt} . \%$, (B) $70 \mathrm{wt} . \%$, (C) $55 \mathrm{wt} . \%$, and (D) $40 \mathrm{wt} . \%$ of tung oil after heating at $100{ }^{\circ} \mathrm{C}$ for $24 \mathrm{~h}$.

From the DSC curves in Figure 6, it can be seen that all samples exhibit an exothermic peak in the $150-160{ }^{\circ} \mathrm{C}$ regardless of the sample composition. Those peaks indicate an exothermic reaction occurring at that temperature, suggesting further crosslink of unreacted carbon-carbon double bonds. The presence of such peak in the DSC curves of all four compositions demonstrates that none of the dried products are fully polymerized when subjected to the cure conditions $\left(100{ }^{\circ} \mathrm{C}, 24 \mathrm{~h}\right)$, revealing a need for a post-cure step after drying. 
Table 1 lists the enthalpy and the temperature for the maximum of the exothermic peaks observed for the samples in Figure 6. The enthalpy, calculated from the integration of the corresponding peaks is highest in the products containing the greatest amount of tung oil. This information suggests that samples with lower amounts of tung oil are polymerized to a higher extent during cure when compared to samples having a higher concentration of tung oil. This is most likely due to the relative reactivity of the oil and the other monomers. Due to its chemical structure, tung oil is expected to be less reactive towards free radicals than DVB or BMA. Therefore, increasing the amount of tung oil in the resin makes it less reactive and a higher portion of the monomers remains unreacted after the cure, generating higher enthalpies upon heating during the DSC experiments.

Table 1. Enthalpy associated to exothermic peak and peak max temperatures for differential scanning calorimetry (DSC) curves of products containing $40 \mathrm{wt} . \%, 55 \mathrm{wt} . \%, 70 \mathrm{wt} . \%$, and $80 \mathrm{wt} . \%$ of tung oil.

\begin{tabular}{ccc}
\hline Tung Oil (wt.\%) & Enthalpy (J/g) & Peak Max $\left({ }^{\circ} \mathbf{C}\right)$ \\
\hline 40 & 50 & 168 \\
55 & 63 & 160 \\
70 & 97 & 155 \\
80 & 99 & 159 \\
\hline
\end{tabular}

When comparing the peak max temperatures in Table 1, there is an apparent trend of decrease in the temperature with increases in the percentage of tung oil in the sample for samples containing up to $70 \mathrm{wt} . \%$ of oil. This behavior could be related to the overall number of carbon-carbon double bonds per monomer molecules. With increasing amounts of tung oil, there is a natural increase in the number of carbon-carbon double bonds that remain unreacted in the resin after cure. With a larger number of carbon-carbon double bonds, it is expected that the residual polymerization during the DSC experiments will start more easily, requiring a lower temperature. This trend, however, doesn't hold true for the sample containing $80 \mathrm{wt} . \%$ of tung oil, which exhibits a slightly higher peak max than the sample with $70 \mathrm{wt} . \%$ of tung oil. At this stage, after repeating these experiments three times and preparing new samples with consistent results, it is unclear why the sample containing $80 \mathrm{wt} . \%$ of tung oil exhibits a different behavior than the other ones. The answer could be in micro-phase separation phenomena happening at a very small scale within the sample, but further investigation would be needed in order to fully understand this behavior. Overall, the DSC results suggest that the sample containing $40 \mathrm{wt} . \%$ of tung oil is the one with the highest extent of polymerization after the $24 \mathrm{~h}$ curing process, but the implementation of a post-cure step is needed for full polymerization of the sample.

In a previous work [13], it had been demonstrated that the bulk polymerization of a resin with the same monomer composition as the sample containing $40 \mathrm{wt} . \%$ of tung oil had been completed after heating at $140{ }^{\circ} \mathrm{C}$ for $2 \mathrm{~h}$ and $50 \mathrm{~min}$. Therefore, in order to obtain a completely cured system, a post-cure step at $140{ }^{\circ} \mathrm{C}$ was implemented here after drying the emulsions. DSC was used to assess any residual cure in the dried emulsions after post-curing at $140{ }^{\circ} \mathrm{C}$ for $1.5 \mathrm{~h}$ and $2 \mathrm{~h}$ (Figure 7).

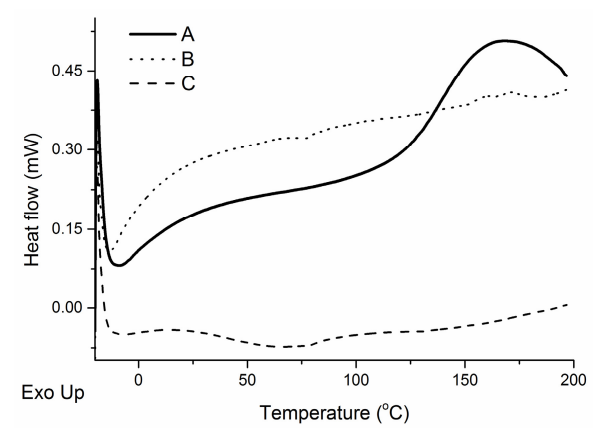

Figure 7. DSC curves of dried emulsions containing $200 \mathrm{mg}$ of total monomer weight with $40 \mathrm{wt} \%$ of tung oil (A) before post-cure, (B) post-cured at $140{ }^{\circ} \mathrm{C}$ for $1.5 \mathrm{~h}$, and (C) post-cured at $140{ }^{\circ} \mathrm{C}$ for $2 \mathrm{~h}$. 
When comparing the DSC curves of samples post-cured for $0 \mathrm{~h}, 1.5 \mathrm{~h}$, and $2 \mathrm{~h}$ (Figure 7), it becomes evident that the exothermic event at approximately $160{ }^{\circ} \mathrm{C}$ decreases with post-cure time. Indeed, the peak is obvious and prominent in the sample that wasn't post-cured (Figure 7A). After a post-cure time of $1.5 \mathrm{~h}$ (Figure 7B), the peak is very subtle. When the sample is subjected to a post-cure of $2 \mathrm{~h}$ (Figure 7C), no peaks can be detected at $160^{\circ} \mathrm{C}$. The appearance of a change in the baseline at approximately $70^{\circ} \mathrm{C}$ for the post-cured samples (Figure 7B,C) may be indicative of a glass transition temperature. The absence of considerable exothermic peaks after a post-cure of $2 \mathrm{~h}$ suggests that no residual cure takes place during the DSC experiment, indicating that the polymerization process is complete. Therefore, a post-cure step of $2 \mathrm{~h}$ at $140{ }^{\circ} \mathrm{C}$ is crucial in order to complete the polymerization of the system.

Raman spectroscopy was also used to verify the completion of the reaction after a $2 \mathrm{~h}$ post-cure at $140{ }^{\circ} \mathrm{C}$ (Figure 8). The spectra corresponding to asolectin, tung oil, and BMA components (Figure 8A-C) exhibit a peak in the $1630-1650 \mathrm{~cm}^{-1}$ range corresponding to $C=C$ streches. The absence of a significant peak in the same region of the spectrum of the post-cured sample (Figure 8D) suggests that the carbon-carbon double bonds in the monomers were completely reacted during the polymerization and post-cure processes. The additional peak observed in the spectrum of BMA at approximately $1725 \mathrm{~cm}^{-1}$ can be attributed to the carbonyl group.

A

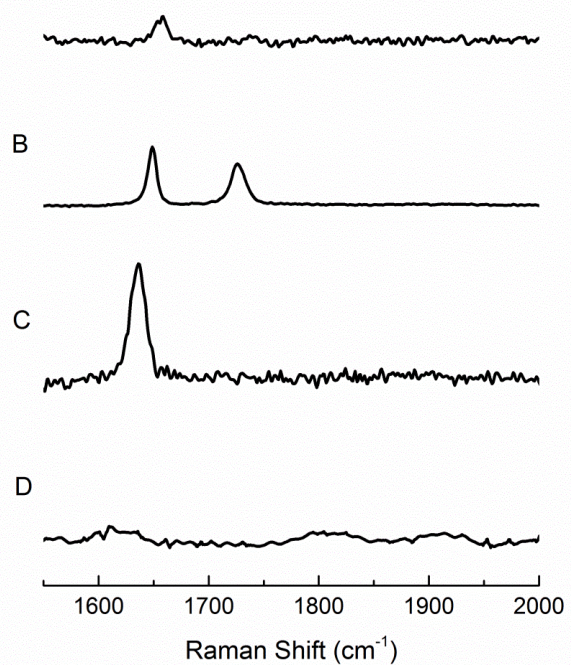

Figure 8. Raman spectra of (A) asolectin, (B) BMA, (C) tung oil, and (D) dried emulsion containing $200 \mathrm{mg}$ of total monomer weight with $40 \mathrm{wt} . \%$ of tung oil post-cured at $140{ }^{\circ} \mathrm{C}$ for $2 \mathrm{~h}$.

Since fully polymerized samples were obtained from the emulsion containing $40 \mathrm{wt} . \%$ tung oil after a post cure step of $2 \mathrm{~h}$ at $140^{\circ} \mathrm{C}$, the post cure step was applied to all monomer compositions. After the post cure step, emulsions containing $40-80 \mathrm{wt} . \%$ of tung oil were analyzed by DSC (Figure 9) and TGA (Figure 10).

The similarity among the DSC curves from samples containing 40-80 wt.\% of tung oil (Figure 9) suggests that a fully cured system can be obtained from any of these compositions after a $2 \mathrm{~h}$ post cure step at $140{ }^{\circ} \mathrm{C}$. Indeed, no obvious exothermic peaks can be observed at approximately $160^{\circ} \mathrm{C}$ after the post-cure (Figure 9). It can also be seen that the change in the baseline at approximately $70{ }^{\circ} \mathrm{C}$, possibly indicating a glass transition temperature, is present in all curves in Figure 9. TGA results (Figure 10) also illustrate similar thermal properties between post-cured samples prepared with $200 \mathrm{mg}$ of total monomer weight and with $40-80 \mathrm{wt} . \%$ of tung oil. Very little variability with no clear trend can be observed among the samples when heated to $600{ }^{\circ} \mathrm{C}$ under air. 


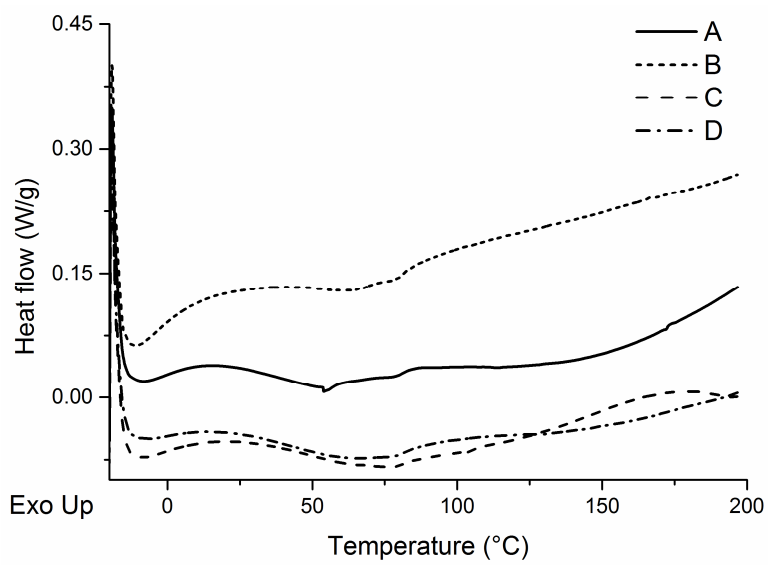

Figure 9. DSC curves of dried latexes containing $200 \mathrm{mg}$ of total monomer weight with (A) $80 \mathrm{wt}$ \% of tung oil, (B) $70 \mathrm{wt} . \%$ of tung oil, (C) $55 \mathrm{wt} . \%$ of tung oil, and (D) $40 \mathrm{wt} . \%$ of tung oil. All samples were subjected to an initial cure step at $100{ }^{\circ} \mathrm{C}$ for $24 \mathrm{~h}$, followed by a drying process in a vacuum oven at $60{ }^{\circ} \mathrm{C}$ for $24 \mathrm{~h}$, and a post cure step at $140{ }^{\circ} \mathrm{C}$ for $2 \mathrm{~h}$.

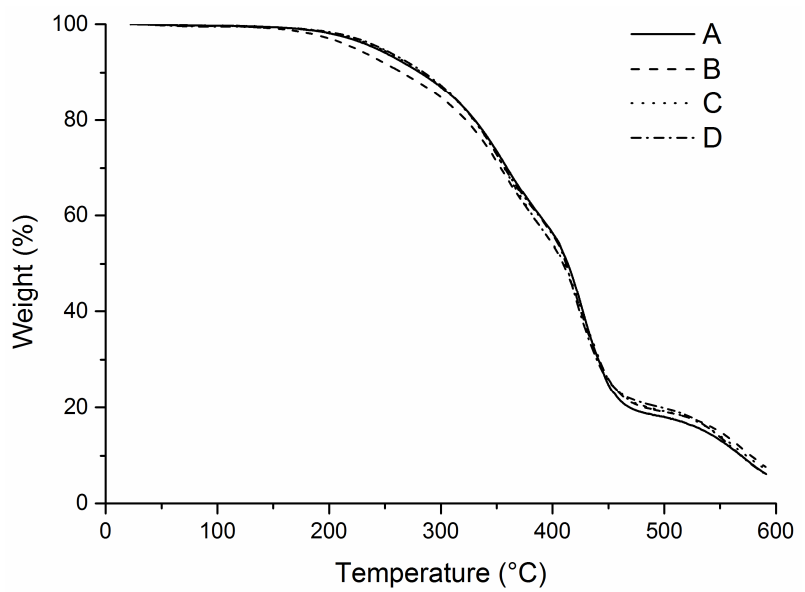

Figure 10. Thermogravimetric Analysis (TGA) curves of dried latexes containing $200 \mathrm{mg}$ of total monomer weight with (A) 80 wt.\% of tung oil, (B) 70 wt.\% of tung oil, (C) 55 wt.\% of tung oil, and (D) $40 \mathrm{wt} . \%$ of tung oil. All samples were subjected to an initial cure step at $100{ }^{\circ} \mathrm{C}$ for $24 \mathrm{~h}$, followed by a drying process in a vacuum oven at $60^{\circ} \mathrm{C}$ for $24 \mathrm{~h}$, and a post cure step at $140{ }^{\circ} \mathrm{C}$ for $2 \mathrm{~h}$.

Transmission microscopy was performed on a cured emulsion (Figure 11), revealing the morphology of the system upon cure. A darker outer layer of the structures seen and a lighter core (more clearly seen in Figure 11A) suggests the formation of hollow spheres, giving the latexes described in this manuscript a range of potential applications besides coatings, including nano-catalyst encapsulation and drug delivery agents. Indeed, upon emulsification in the presence of asolectin, droplets of non-polar monomers are formed. The crosslinking of the droplets' contents during polymerization ensures that the droplet morphology is maintained even after solvent removal, as confirmed by STEM (Figure 11). Due to the low monomer concentration in the emulsions originally prepared, it is assumed that most of the original volume of a droplet consists of xylene, which is removed upon drying, leaving a hollow sphere referred to as a "vesicle".

Despite a few larger particles seen in Figure 11A, the majority of the particles exhibit diameters on the order of $100 \mathrm{~nm}$ (Figure 11B,C). In order to more accurately determine the vesicles' particle size, cured emulsions were characterized using a zetasizer. Table 2 displays average diameter and polydispersity data for samples containing $200 \mathrm{mg}$ of total monomer weight and $40-80 \mathrm{wt} . \%$ of tung oil. 


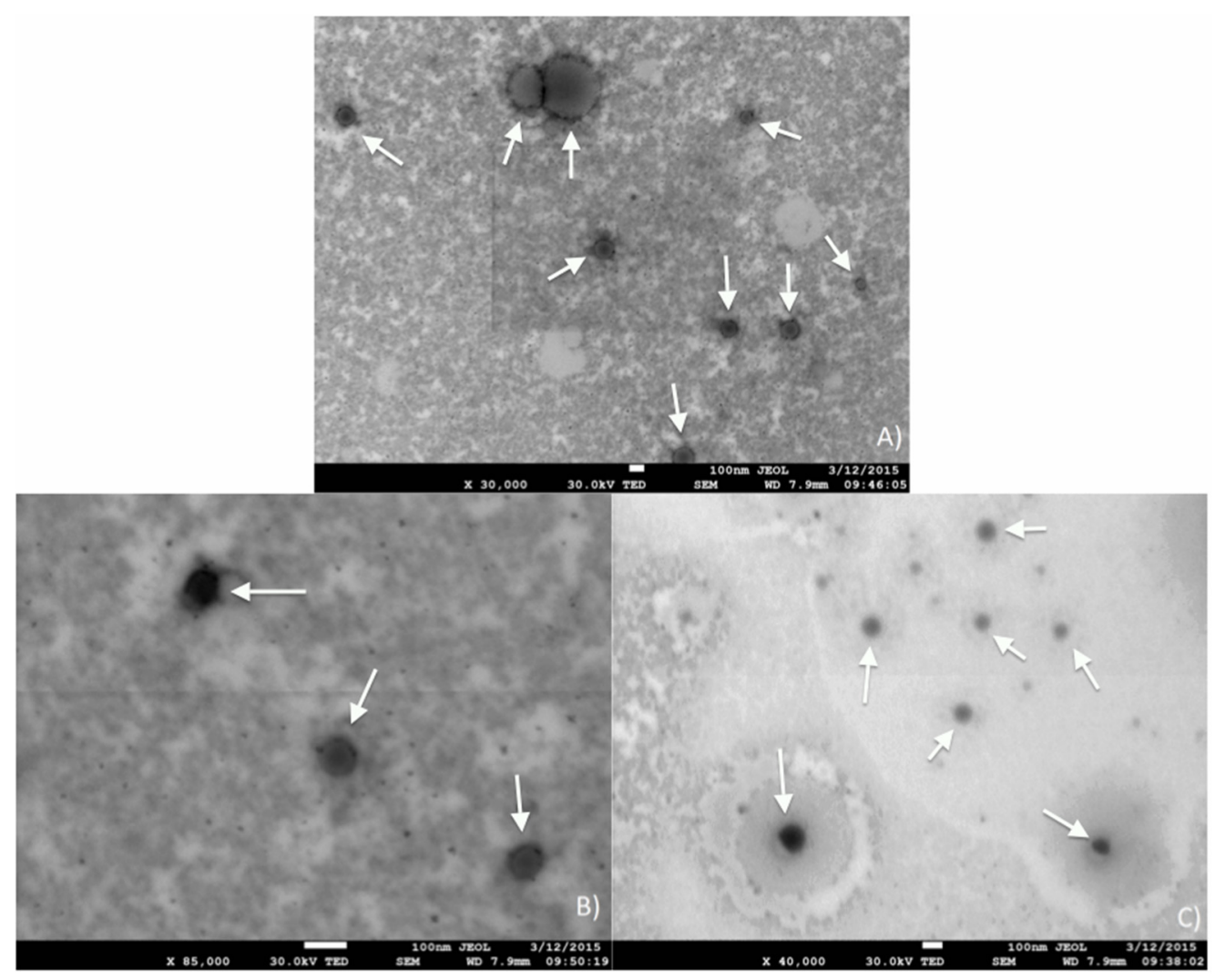

Figure 11. STEM images of a cured sample suspension containing $200 \mathrm{mg}$ of total monomer weight with 40 wt.\% of tung oil at (A) 30,000 $\times$ magnification, (B) 85,000 $\times$ magnification, and (C) 40,000 $\times$ magnification. All scale bars correspond to $100 \mathrm{~nm}$, while white arrows indicate vesicles.

Table 2. Zetasizer results for cured emulsions containing $200 \mathrm{mg}$ of total monomer weight with $40-80$ wt.\% of tung oil.

\begin{tabular}{cccc}
\hline Entry & Tung Oil (wt.\%) & Average Diameter (nm) & PDI \\
\hline 1 & 40 & 170.4 & 0.278 \\
2 & 55 & 177.0 & 0.192 \\
3 & 70 & 185.0 & 0.363 \\
4 & 80 & 219.0 & 0.251 \\
\hline
\end{tabular}

From the data in Table 2, a clear trend of increasing particle size can be observed for increasing tung oil content. Such behavior allows for some level of control on the particle average diameter by simply adjusting the content of tung oil in the monomer mixture. Such aspect confers versatility to the system presented, since it is possible to design vesicles of desired size depending on the targeted application. The average diameter range obtained (170-219 nm, Table 2) is ideal for the encapsulation of nanomaterials. The polydispersity index (PDI) related to vesicle diameter ranges from 0.2 to 0.4 , indicating a reasonably, but consistent broad distribution of particle sizes, with no clear connections with the oil content in the monomer mixture. Despite its polydisperse nature, the emulsions have shown good stability, as previously mentioned in the text, and as observed in similar systems [20].

\section{Conclusions}

Due to the current environmental challenges faced by mankind, the development of sustainable systems that rely more heavily in biorenewable chemicals and materials has become a strategic research focus area. Within that context, the present manuscript describes the preparation of 
bio-based thermosetting vesicles from tung oil and asolectin, a mixture of phospholipids obtained from soybeans. The system consists of a xylene/water emulsion containing tung oil, BMA, DVB, and asolectin as comonomers. Because of its amphiphilic nature, asolectin works as a natural surfactant that is crosslinked with the other co-monomers in the system upon free radical polymerization. After optimizing the cure conditions for the system and the comonomer composition, completeness of polymerization reaction was confirmed by DSC experiments and formation of vesicles was verified via STEM. Study of the vesicle size distribution through Zetasizer measurements revealed that vesicles had an average diameter of $163.5 \mathrm{~nm}$, with a PDI of 0.206 , configuring stable vesicles with potential for the encapsulation of nanomaterials.

Acknowledgments: The authors would like the acknowledge Marshall Ming for sharing his experience in the field of emulsion polymerization at the early stages of this project. The authors are also indebted to the Chemistry Department and the College of Science and Mathematics at Georgia Southern University for the infrastructure and financial support provided in many instances for the duration of this project.

Author Contributions: Rafael L. Quirino conceived and designed the experiments; Ashley Johns performed the synthesis, the Maldi-TOF, the DEA, the DSC, the Raman spectroscopy, and the TGA experiments; Kyle Edwards performed the STEM experiments, Sarah Inglesby performed the Zetasizer experiments, Ashley Johns analyzed all data; Rafael L. Quirino provided reagents/materials/analysis tools; and Ashley Johns and Rafael L. Quirino wrote the paper.

Conflicts of Interest: The authors declare no conflict of interest.

\section{References}

1. Valverde, M.; Andjelkovic, D.; Kundu, P.P.; Larock, R.C. Conjugated low-saturation soybean oil thermosets: Free-Radical copolymerization with dicyclopentadiene and divinylbenzene. J. Appl. Polym. Sci. 2008, 107, 423-430. [CrossRef]

2. Li, F.K.; Larock, R.C. Thermosetting polymers from cationic copolymerization of tung oil: Synthesis and characterization. J. Appl. Polym. Sci. 2000, 78, 1044-1056. [CrossRef]

3. Quirino, R.L.; Woodford, J.; Larock, R.C. Soybean and linseed oil-based composites reinforced with wood flour and wood fibers. J. Appl. Polym. Sci. 2012, 124, 1520-1528. [CrossRef]

4. Pfister, D.P.; Larock, R.C. Cationically cured natural oil-based green composites: Effect of the natural oil and the agricultural fiber. J. Appl. Polym. Sci. 2012, 123, 1392-1400. [CrossRef]

5. Xia, Y.; Larock, R.C. Castor oil-based thermosets with varied crosslink densities prepared by ring-opening metathesis polymerization (ROMP). Polymer 2010, 51, 2508-2514. [CrossRef]

6. Bonnaillie, L.M.; Wool, R.P. Thermosetting foam with a high bio-based content from acrylated epoxidized soybean oil and carbon dioxide. J. Appl. Polym. Sci. 2007, 105, 1042-1052. [CrossRef]

7. Sharma, H.O.; Alam, M.; Riaz, U.; Ahmad, S.; Ashraf, S.M. Miscibility studies of polyesteramides of linseed oil and dehydrated castor oil with poly(vinyl alcohol). Int. J. Polym. Mater. Polym. Biomater. 2007, 56, 437-451. [CrossRef]

8. Zhang, G.; Zhao, L.; Hu, S.; Gan, W.; Yu, Y.; Tang, X. A novel biobased resin-epoxidized soybean oil modified cyanate ester. Polym. Eng. Sci. 2008, 48, 1322-1328. [CrossRef]

9. Liu, C.; Yang, X.; Cui, J.; Zhou, Y.; Hu, L.; Zhang, M.; Liu, H. Tung oil based monomer for thermosetting polymers: Synthesis, characterization, and copolymerization with styrene. BioResources 2012, 7, 447-463.

10. De Espinosa, L.M.; Meier, M. Plant oils: The perfect renewable resource for polymer science? Eur. Polym. J. 2011, 47, 837-847. [CrossRef]

11. Pfister, D.P.; Baker, J.R.; Henna, P.H.; Lu, Y.S.; Larock, R.C. Preparation and properties of tung oil-based composites using spent germ as a natural filler. J. Appl. Polym. Sci. 2008, 108, 3618-3625. [CrossRef]

12. Quirino, R.L.; Larock, R.C. Rice hull biocomposites. I. Preparation of a linseed oil-based resin reinforced with rice hulls. J. Appl. Polym. Sci. 2011, 121, 2039-2049. [CrossRef]

13. Johns, A.; Morris, S.; Edwards, K.; Quirino, R.L. Asolectin from soybeans as a natural compatibilizer for cellulose-reinforced biocomposites from tung oil. J. Appl. Polym. Sci. 2015, 132, 41833-41842. [CrossRef]

14. Navratil, T.; Sestakova, I.; Marecek, V. Supported phospholipid membranes formation at a gel electrode and transport of divalent cations across them. Int. J. Electrochem. Sci. 2011, 6, 6032-6046. 
15. Lu, Y.; Larock, R.C. Soybean oil-based waterborne polyurethane dispersions: Effects of polyol functionality and hard segment content on properties. Biomacromolecules 2008, 9, 3332-3340. [CrossRef] [PubMed]

16. Lu, Y.; Larock, R.C. Aqueous cationic polyurethane dispersions from vegetable oils. ChemSusChem 2010, 3, 329-333. [CrossRef] [PubMed]

17. Lu, Y.; Larock, R.C. Synthesis and properties of grafted latices from a soybean oil-based waterborne polyurethane and acrylics. J. Appl. Polym. Sci. 2011, 119, 3305-3314. [CrossRef]

18. Lu, Y.; Larock, R.C. New hybrid latexes from a soybean oil-based waterborne polyurethane and acrylics via emulsion polymerization. Biomacromolecules 2007, 8, 3108-3114. [CrossRef] [PubMed]

19. Lu, Y.; Xia, Y.; Larock, R.C. Surfactant-free core-shell hybrid latexes from soybean oil-based waterborne polyurethanes and poly(styrene-butyl acrylate). Prog. Org. Coat. 2011, 71, 336-342. [CrossRef]

20. Zhang, Q.; Galvan-Miyoshi, J.M.; Pezzotti, F.; Ming, W. Synthesis and surface properties of PDMS-containing latexes by emulsion polymerization using AIBN as the initiator. Eur. Polym. J. 2013, 49, 2327-2333. [CrossRef]

(C) 2016 by the authors; licensee MDPI, Basel, Switzerland. This article is an open access article distributed under the terms and conditions of the Creative Commons Attribution (CC-BY) license (http://creativecommons.org/licenses/by/4.0/). 